\title{
ASSOCIAÇÃO ENTRE ÍNDICES ANTROPOMÉTRICOS E MARCADORES DE RISCO CARDIOVASCULAR EM ADOLESCENTES
}

Association between anthropometric indices and cardiovascular risk markers in adolescents

Associación entre índices antropométricos y marcadores de riesgo cardiovascular en adolescentes

\author{
Adênila Araújo Cavalcante 1 , Rafaella Lemos Alves ${ }^{2}$, Lara Camargo \\ Santos $^{3}$, Sylvia do Carmo Castro Franceschini ${ }^{4}$, Silvia Eloiza Priore ${ }^{4}$, \\ Kellen Cristine Silva ${ }^{5 *}$ \\ ${ }^{1}$ Nutricionista, Palmas, Brasil. \\ ${ }^{2}$ Curso de Nutrição, Faculdade LS Educacional, Brasília, Brasil. \\ ${ }^{3}$ Instituto Brasiliense de Nutrologia, Brasília, Brasil. \\ ${ }^{4}$ Departamento de Nutrição e Saúde, Universidade Federal de Viçosa, Viçosa, Brazil. \\ ${ }^{5}$ Curso de Nutrição, Universidade Federal do Tocantins, Palmas, Brasil.
}

*Correspondência: Complexo Laboratorial de Nutrição e Saúde, Universidade Federal do Tocantins, Av. NS 15, 109 Norte, Palmas, Tocantins, Brasil. CEP:77.010-090. e-mail

kellensilva@uft.edu.br

Artigo recebido em 10/04/2020 aprovado em 27/08/2020 publicado em 30/10/2020.

\section{RESUMO}

A antropometria tem sido utilizada para rastrear risco de desenvolvimento de doenças cardiovasculares, e quanto mais precocemente este risco for detectado, maiores as chances de diminuir a ocorrência de ataques cardíacos e infarto na vida adulta e idosa. Portanto, o objetivo desse estudo foi identificar a associação entre medidas e índices antropométricos e marcadores de risco cardiovascular em adolescentes. A população do estudo foi selecionada por conveniência e totalizou 72 sujeitos de 10 a 19 anos. As medidas e índices antropométricos utilizados foram: índice de massa corporal (IMC), perímetro da cintura (PC), razão cintura-estatura (RCE) e perímetro do pescoço (PP). Pressão arterial, glicemia e perfil lipídico constituíram os marcadores de risco cardiovascular. Para identificar a associação entre as variáveis utilizou-se o Teste do Qui-quadrado e Exato de Fisher. Observou-se que $22 \%$ dos adolescentes apresentaram IMC elevado, 18,1\% PC elevado, 13,5\% RCE maior que 0,5 e $83 \%$ PP acima do recomendado. IMC, PC e RCE foram associados à pressão arterial e colesterol total elevados. Conclui-se que medidas e índices antropométricos estão associados à marcadores de risco cardiovascular na adolescência. Portanto, a antropometria pode ser uma ferramenta útil no rastreamento de indivíduos com risco para o desenvolvimento de doenças cardiovasculares precocemente.

Palavras-chave: antropometria; adolescência; doenças cardiovasculares.

\section{ABSTRACT}

Anthropometry has been used to track risk of developing cardiovascular disease and the earlier risk is detected, the greater chances of decreasing heart attacks and infarction in adults and elderly. Therefore, aim of this study was to identify association between anthropometric measures and indices and cardiovascular risk markers in adolescents. Study population was selected for convenience and enrolled 72 subjects ages 10-19 years). Anthropometric measures and indices used were: body mass index (BMI), waist circumference (WC), waist-height ratio (WHR) and neck 
circumference (NC). Blood pressure, blood glucose and lipid profile were cardiovascular risk markers. To identify the association between the variables, Chi-square test and Fisher's exact test were used. We observed that 22\% of the adolescents had high BMI, 18.1\% high WC, 13.5\% WHR greater than 0.5 and $83 \%$ NC above the recommended. BMI, WC and WHR were associated with high blood pressure and total cholesterol. We concluded that anthropometric measures and indexes are associated with cardiovascular risk markers in adolescence. Therefore, anthropometry can be a useful tool in screening individuals at risk for development of cardiovascular diseases early. Keywords: anthropometry; adolescence; cardiovascular diseases.

\section{RESUMEN}

La antropometría ha sido utilizada para rastrear el riesgo de desarrollo de enfermedades cardiovasculares y cuanto más precocemente este riesgo detectado, mayor las posibilidades de disminuir una ocorrência de ataques cardíacos e infarto em adultos e idosos. Por lo tanto, el objetivo de este estudio fue identificar la asociación entre las medidas y índices antropométricos y los marcadores de riesgo cardiovascular en adolescentes. La población de estudio fue seleccionada por conveniencia y totalizó 72 sujetos de 10 a 19 años. Las medidas e índices antropométricos utilizados fueron: índice de masa corporal (IMC), circunferencia de la cintura (CCa), relación cintura-altura (RCA) y circunferencia del cuello (CCo). La presión arterial, la glucosa en sangre y el perfil lipídico fueron los marcadores de riesgo cardiovascular. Para identificar la asociación entre las variables, se utilizaron la prueba de Chi-cuadrado y la prueba exacta de Fisher. Se observó que el $22 \%$ de los adolescentes tenían un IMC elevado, $18.1 \%$ de CCa alto, 13.5\% RCA mayor que 0.5 y 83\% CCo por encima de lo recomendado. IMC, CCa y RCA se asociaron con presión arterial alta y colesterol total. Se concluye que las medidas índices antropométricos están asociados con marcadores de riesgo cardiovascular en la adolescencia. Por lo tanto, la antropometría puede ser una herramienta útil en la detección temprana de personas en riesgo de desarrollar enfermedades cardiovasculares.

Descriptores: antropometria; adolescencia; enfermedades cardiovasculares.

\section{INTRODUÇÃO}

Baseado na patogênese das doenças cardiovasculares, sabe-se que os desfechos clínicos ocorrem predominantemente na idade adulta e idosa, no entanto as evidências sustentam que os estágios iniciais da doença iniciam na infância e adolescência (BERENSON et al., 1989; STRONG; MCGILL, 1969). Marcadores de risco cardiovascular são amplamente reportados em estudos que analisam populações jovens. No Brasil, foi realizado o Estudo de Riscos Cardiovasculares em Adolescentes (ERICA) que estimou a prevalência de obesidade, dislipidemia, hipertensão, diabetes mellitus e síndrome metabólica em 75.000 adolescentes vivendo nas capitais e em cidades com mais de 100 mil habitantes. As alterações mais prevalentes foram lipoproteína de alta densidade baixa, seguida de hipercolesterolemia e sobrepeso (BLOCH et al., 2016; FARIA-NETO et al., 2016).
A Organização Mundial da Saúde atribui à identificação do risco cardiovascular na infância e adolescência com uma das estratégias de controle do avanço das doenças cardiovasculares em idades mais avançadas. Quanto mais cedo estes fatores forem detectados maior a chance de impedir ou retardar a ocorrência de um evento cardiovascular (WHO, 2013). Portanto, a adolescência é uma fase da vida em que o risco cardiovascular é eminente e precisa ser rastreado para que medidas de promoção, prevenção e tratamento sejam implementadas o mais precocemente possível.

Para isso a utilização de métodos práticos e confiáveis de rastreamento de marcadores de risco cardiovascular podem acelerar a detecção de indivíduos potencialmente de risco. Neste sentido, as medidas e índices antropométricos podem ser úteis, como o índice de massa corporal (IMC), pois apresentam inúmeras vantagens operacionais, baixo 
custo e correlaciona-se bem com a gordura corporal total (FERNANDES et al., 2007).

Outras medidas como a razão cintura estatura (RCE) e perímetro da cintura (PC) são úteis para identificar indivíduos com alto risco metabólico e cardiovascular, pois a inadequada distribuição de adiposidade é apontada como fator de risco, sobretudo naqueles com excesso de peso. Adolescentes com perímetro da cintura elevado possuem valores significantemente maiores de triglicerídeos, insulina, HOMA-IR, leptina, pressão arterial sistólica e diastólica (PEREIRA et al., 2011). Moreira et al., observaram em uma amostra de adolescentes de 15 a 18 anos que o IMC, RCE e PC estão positiva e significativamente associadas ao risco cardiometabólico em ambos os sexos. Diferentes índices e medidas antropométricos apresentaram bom desempenho na detecção alto risco cardiomatabólico, na adolescência (MOREIRA et al., 2011; SILVA et al., 2020).

$\mathrm{O}$ perímetro do pescoço (PP) é outra medida proposta como preditora do risco cardiovascular. Sua utilização está baseada no fato que o PP é capaz de refletir a gordura subcutânea do tronco superior, e ainda relaciona-se tanto com a gordura visceral abdominal quanto com marcadores de risco cardiometabólico (PREIS et al., 2010). Estudos realizados na adolescência observaram que as associações para esta medida são comparáveis àquelas encontradas para o IMC, PC e RCE e perímetro o quadril (ANDROUTSOS et al., 2012; GOMEZ-ARBELAEZ et al., 2016; KURTOGLU et al., 2012).

Diante disso, o objetivo deste estudo foi avaliar a associação entre medidas e índices antropométricos e o risco cardiovascular entre adolescentes de uma escola da cidade de Palmas (TO).

\section{MATERIAIS E MÉTODOS}

\section{Delineamento do estudo}

O presente trabalho foi realizado com a população do estudo piloto do projeto de doutoramento intitulado "Medidas antropométricas e estilo de vida associados ao risco cardiovascular nas três fases da adolescência". O estudo original é de do tipo transversal de base escolar, descritivo e analítico, cuja unidade de estudo é o indivíduo (PAGANO, 2008).

Para a realização do estudo, uma escola pública da região central da cidade de Palmas (TO) foi selecionada por conveniência. O cálculo amostral do projeto supracitado totalizou 732 sujeitos, portanto o estudo piloto correspondeu a $10 \%$ da amostra, totalizando 74 adolescentes. A seleção dos indivíduos foi realizada por sorteio no universo de alunos que estavam cursando o $6^{\circ}$ ano do Ensino Fundamental (EF) até o $3^{\circ}$ ano do Ensino Médio (EM).

Foram incluídos adolescentes de 10 a 19 anos 11 meses e 29 dias, cujos pais e/ou responsáveis permitiram sua participação no estudo e que o adolescente também aceitasse participar, no caso daqueles menores de 18 anos. Para os maiores de 18 anos, foram incluídos aqueles que assinaram o TCLE. Exclui-se adolescentes que não completaram todas as etapas do estudo, relataram ter alguma restrição na dieta (ex: fenilcetunúria, diabetes mellitus, doença celíaca), que faziam uso regular de medicamentos que alterassem a pressão arterial, o metabolismo lipídico e glicídico e adolescentes grávidas e puérperas.

A coleta dos dados foi realizada na própria escola, em local destinado exclusivamente para este fim, de forma a minimizar qualquer desconforto que pudesse ocorrer ao adolescente. Para padronizar os métodos propostos na pesquisa, foi realizado um treinamento e calibração das medidas antropométricas com os avaliadores antes do início da coleta de dados. 


\section{Medidas e índices antropométricos}

Peso e altura foram coletados conforme a Norma Técnica do Sistema de Vigilância Alimentar e Nutricional (SISVAN, 2004). O peso foi aferido utilizando balança digital eletrônica Marte ${ }^{\circledR}$ com capacidade de $200 \mathrm{~kg}$ e precisão de $0,1 \mathrm{~kg}$ e a estatura

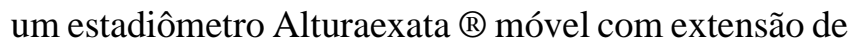
2,13m e precisão de $1 \mathrm{~mm}$.

$\mathrm{O}$ estado nutricional dos adolescentes foi identificado a partir do Índice de Massa Corporal para Idade (IMC/I) e classificado conforme proposta da Organização Mundial da Saúde (ONIS et al., 2007). Foram utilizados os seguintes pontos de corte: < escore- $\mathrm{z}-2=$ magreza, $\geq$ escore- $\mathrm{z}-2$ e $\leq$ escore $+1=$ eutrofia, $>$ escore- $z+1$ e $\leq$ escore- $z+2=$ sobrepeso, $>$ escore- $\mathrm{Z}+2=$ obesidade. Para a compilação desses dados antropométricos foi utilizado o software WHO Antro plus 2009, versão 3. Nas análises dos dados, magreza e eutrofia foram agrupados como peso adequado e sobrepeso e obesidade como excesso de peso.

As perimetrias foram realizadas utilizando uma fita métrica inelástica e flexível com extensão de 1,5m e precisão de $0,5 \mathrm{~cm}$. O perímetro da cintura foi aferido na menor cintura abdominal entre a última costela e a crista ilíaca anterior superior (JELLIFE, 1968). Foram realizadas três medidas em cada ponto anatômico e o valor médio utilizados nas análises. Quando a diferença entre elas foi maior que $1 \mathrm{~cm}$, realizou-se a terceira medida até que a defasagem fosse aceitável. O percentil 80 de acordo com o sexo da população foi calculado, aqueles adolescentes que estavam acima ou igual a este percentil foram classificados com risco para doenças cardiovasculares.

A partir da divisão da medida da cintura pela estatura, foi derivada a razão cintura-estatura (RCE). Valores iguais ou maiores que 0,5 foram considerados como risco para doenças cardiovasculares elevados (BROWNING; HSIEH; ASHWELL, 2010).

$\mathrm{O}$ perímetro do pescoço $(\mathrm{PP})$ foi na região mediana entre a coluna cervical média e o pescoço médio-anterior. Em homens com proeminência laríngea aparente (pomo de Adão), a medida foi obtida logo abaixo desta. Foram realizadas três medidas e o valor médio foi utilizado nas análises. Quando a diferença entre elas foi maior que $1 \mathrm{~cm}$, realizou-se terceira medida até que a defasagem fosse aceitável. O ponto de corte para identificação do risco cardiovascular foi $30 \mathrm{~cm}$ para meninos e $29 \mathrm{~cm}$ para meninas (GOMEZ-ARBELAEZ et al., 2016).

\section{Marcadores de risco cardiovascular}

Dosagens de colesterol total (CT), lipoproteína de baixa densidade (LDL), lipoproteína de alta densidade (HDL), Triglicerídeos (TG) e glicemia foram determinadas na amostra de sangue dos participantes. Para a realização dessas dosagens, os adolescentes estavam em jejum de 12 horas, foram coletados $12 \mathrm{~mL}$ de sangue e a coleta foi realizada pela equipe de um Laboratório de Análises Clínicas da cidade de Palmas.

As concentrações séricas de CT, HDL, e TG foram analisadas por fotometria de absorção pelo método enzimático. A determinação do LDL foi feita pela fórmula de Friedewald, observando- se as limitações para a metodologia (TG>400 mg/dL). Os níveis séricos de CT e frações foram classificados conforme a Atualização da Diretriz Brasileira de Prevenção Cardiovascular, considerando os seguintes pontos de corte: CT $<170 \mathrm{mg} / \mathrm{dL}$, HDL $>45 \mathrm{mg} / \mathrm{dL}$, LDL $<110 \mathrm{mg} / \mathrm{dL}$, TG $(0-9$ anos $)<75 \mathrm{mg} / \mathrm{dL}, \mathrm{TG}(10$ - 19 anos) <90 mg/dL (SOCIEDADE BRASILEIRA DE CARDIOLOGIA (SBC), 2017). 
A glicose sérica foi medida pelo método colorimétrico e classificada como glicemia normal valores $<100 \mathrm{mg} / \mathrm{dL}$, tolerância à glicose diminuída $\geq$ $100 \mathrm{mg} / \mathrm{dL}$ e $<126 \mathrm{mg} / \mathrm{dL}$ e elevada $\geq 126 \mathrm{mg} / \mathrm{dL}$, conforme recomendado pela Sociedade Brasileira de Diabetes (SOCIEDADE BRASILEIRA DE DIABETES (SBD), 2018).

A avaliação clínica consistiu da medida da pressão arterial (PA). A aferição foi realizada no braço esquerdo depois de um período mínimo de descanso de 15 minutos, em duplicata, com intervaldo de 1 minuto entre elas e o utilizou-se a média dos valores. Utilizouse um monitor de pressão sanguínea de inflação automática da marca OMROM®, modelo HEM 705$\mathrm{CP}$ e manguitos com largura e comprimento proporcionais ao perímetro do braço do adolescente (proporção largura/comprimento de 1:2). A classificação foi feita conforme o sexo e o percentil de estatuta-para-idade (SOCIEDADE BRASILEIRA DE CARDIOLOGIA (SBC), 2017). Considerou-se hipertensão arterial quando a PA fosse $\geq$ percentil 90 para indivíduos de 10 a 17 anos para idade e sexo, e PA > 130/85 mmHg para aqueles de 18 a 19 anos.

\section{Análises estatísticas}

O banco de dados foi construído utilizando o Excel versão 2010 e as análises estatísticas foram realizadas utilizando o Statical Package for Social Sciences-SPSS versão 20.0.

As variáveis foram expressas em frequências absoluta e relativa e o teste do Qui-quadrado e Exato de Fisher foram utilizados para verificar as associações. Para todas as análises foi adotado como nível de significância $\alpha=0,05$.

\section{Questões éticas}

A pesquisa foi submetida ao Comitê de Ética e Pesquisa com Seres Humanos da Universidade Federal de Viçosa (protocolo ${ }^{\circ}$ 2.205.399). A coleta de dados foi iniciada somente após a aprovação por este Comitê, quando os pais e/ou responsáveis e adolescentes maiores de 18 anos assinarem o Termo de Consentimento Livre e Esclarecido e os menores de 18 anos assinarem o Termo de Assentimento.

\section{RESULTADOS E DISCUSSÃO}

Do universo de 74 adolescentes selecionados, 2 foram excluídos, pois não completaram todas as etapas do estudo. Assim 72 adolescentes foram avaliados, sendo $51,4 \%(\mathrm{n}=37)$ do sexo masculino e observou-se maior proporção de adolescentes na fase intermediária (Tabela 1).

Tabela 1 - Distribuição da população segundo fase da adolescência e sexo (n=72). Palmas, TO, Brasil, 2017.

\begin{tabular}{lcccccc}
\hline \multirow{2}{*}{ População } & \multicolumn{5}{c}{ Fase da adolescência } \\
\cline { 2 - 7 } & $\begin{array}{c}\text { Fase } \\
\text { inicial } \\
(\mathbf{1 1 - 1 3} \\
\text { anos) }\end{array}$ & $\begin{array}{c}\text { Fase } \\
\text { intermediária } \\
(\mathbf{1 4 - 1 6} \text { anos })\end{array}$ & $\begin{array}{l}\text { Fase } \\
\text { final } \\
(\mathbf{1 7 - 1 9} \\
\text { anos })\end{array}$ \\
\hline & $\mathrm{n}$ & $\%$ & $\mathrm{n}$ & $\%$ & $\mathrm{n}$ & $\%$ \\
\hline Meninos & 15 & 20,8 & 10 & 13,9 & 12 & 16,7 \\
Meninas & 10 & 13,9 & 18 & 25,0 & 7 & 9,7 \\
Total & 25 & 34,7 & 28 & 38,9 & 19 & 26,4 \\
\hline
\end{tabular}

A partir dos dados apresentados na Tabela 2 observa-se que entre os índices antropométricos com maior percentual de inadequação, o primeiro é o PP (83,0\%), seguido do excesso de peso $(22,2 \%)$, PC elevado $(18,1 \%)$ e RCE elevada (13,5\%). Não foi encontrada diferenças entre os sexos.

Em relação aos marcadores de risco cardiovascular, HDL baixo, CT e TG elevados atingiram mais de 30\% dos adolescentes, com destaque para o HDL baixo que está presente em quase a metade deles $(44,2 \%)$. Na sequência, encontramos que o LDL 
e PA elevados foram detectados em $20,9 \%$ e $13,0 \%$ da amostra, respectivamente. O marcador de risco cardiovascular menos prevalente na amostra é a meninos com níveis pressóricos insatisfatórios é 3,6 vezes maior quando comparados às meninas $(p=0,034)$ (Tabela 2).

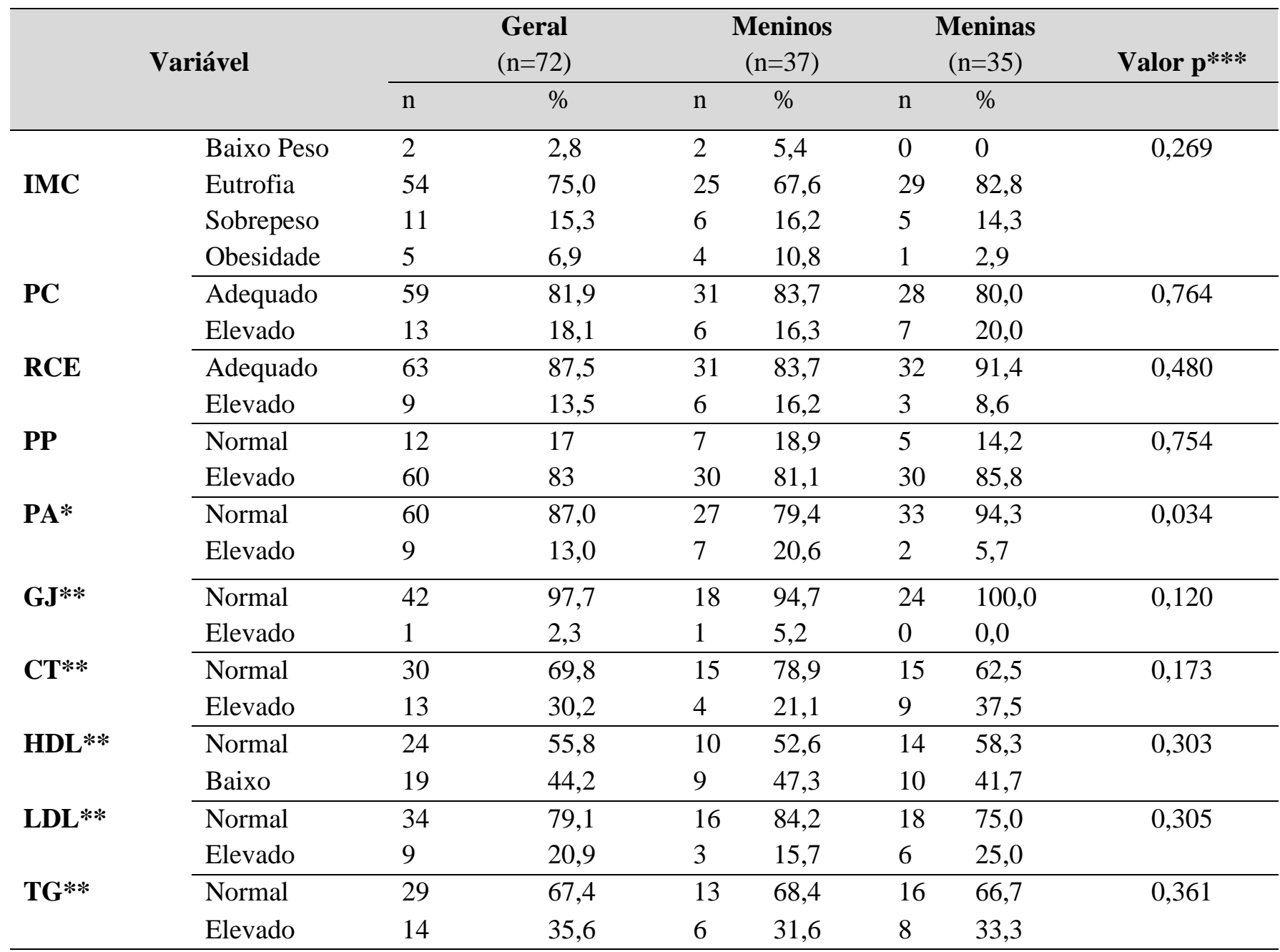

glicemia elevada. Atenta-se para as diferenças de PA elevada entre os sexos, uma vez que a proporção de

Tabela 2 - Índices antropométricos, perfil lipídico, glicêmico e pressórico dos adolescentes segundo o sexo (n=72). Palmas, TO, Brasil, 2017

$* \mathrm{n}=69, * * \mathrm{n}=43, * * *$ Teste do Qui-quadrado ou Exato de Fisher.

IMC: índice de massa corporal; PC: perímetro da cintura; RCE: razão cintura-estatura; PP: perímetro do pescoço; PA: pressão arterial; GJ: glicemia de jejum; CT: colesterol total; HDL: lipoproteínas de alta densidade; LDL: lipoproteínas de baixa densidade; TG: triglicerídeos.

O perfil nutricional e de marcadores do risco cardiovascular nesta população assemelha-se aos resultados encontrados pelo Estudo de Riscos Cardiovasculares em Adolescentes (ERICA), no qual os marcadores de risco cardiovascular mais prevalente são o HDL baixo $(46,8 \%)$ e CT elevado $(20,1 \%)$
(FARIA-NETO et al., 2016). Diversos fatores estão associados à dislipidemia na adolescência, como a baixa escolaridade materna e pertencer a classe socioeconômica $\mathrm{C}$ e $\mathrm{D}$, excesso de peso, consumo de alimentos com alto teor de gorduras totais, trans e 
colesterol e sedentarismo (NETO et al., 2012; RIBAS; DA SILVA, 2014).

A pressão arterial elevada também foi evidenciada em outros estudos como um marcador de risco cardiovascular nessa fase da vida e as prevalências variam entre 11,2\% a 15,8\%. Baixa escolaridade materna, excesso de peso, padrão de consumo de alimentos de risco como salgadinhos e salgados fritos, açúcar, sorvete e achocolatado (FERREIRA; AYDOS, 2010; PINTO et al., 2011; SILVA et al., 2013; VIEIRA et al., 2009). Desta forma, observa-se que condições socioeconômicas e o estilo de vida dos adolescentes são aspectos a serem considerados no controle das doenças cardiovasculares.

$\mathrm{Na}$ tabela 3 estão apresentados os resultados das associações observadas entre índices antropométricos e marcadores de risco cardiovascular. Observou-se que IMC classificado como excesso de peso está associado à PA elevada $(\mathrm{p}=0,026)$ e colesterol total elevado $(\mathrm{p}=0,042)$. Além disso, PC e RCE elevados também estão associados ao colesterol total elevado $(p=0,009 ; p=0,013$, respectivamente). $O$ PP não apresentou associação com nenhum dos marcadores de risco cardiovascular. Glicemia, LDL e TG elevados e HDL baixos não apresentaram associação com nenhuma medida ou índice antropométrico.

Dentre os estudos citados anteriormente, todos identificaram que o excesso de peso medido pelo IMC está associado com algum marcador de risco cardiovascular. Isto decorre do fato que o IMC possui alta correlação com o tecido adiposo total, o qual exerce papel relevante na secreção de mediadores inflamatórios e humorais, que podem favorecer o aumento da pressão arterial e resistência insulínica, e ainda alterar o metabolismo das lipoproteínas desencadeando as dislipidemias (KLEIN et al., 2007; RASK-MADSEN; KAHN, 2012).

Perímetro da cintura e RCE relacionam-se com a concentração de tecido adiposo na região abdominal, apresentando maior sensibilidade a quantidade de tecido adiposo visceral, ou seja, a gordura depositada entre os órgãos (KLEIN et al., 2007). Estudos mostram que esta gordura apresenta maior correlação com o desenvolvimento de diabetes, dislipidemia e hipertensão arterial que o IMC, por potencializar os mecanismos envolvidos na patogênese dessas doenças (KLEIN et al., 2007; WANG et al., 2005).

Apesar dessas diferenças, diversos estudos identificaram que IMC, PC e RCE apresentam similar capacidade de rastreamento de marcadores de risco cardiovascular me adolescentes (BAUER et al., 2015; CHOI et al., 2017; SILVA, et al., 2020). Destaca-se a utilidade RCE, uma vez que sua interpretação dispensa tabelas e gráficos por sexo e idade, além disso, a obtenção das medidas é rápida e de baixo custo (LO et al., 2016).

Revisão de literatura realizada por Magalhães et al., (2014) reuniu estudos que utilizaram PC, RCE e PP como indicador de obesidade central em crianças e adolescentes e identificaram que o PP ainda é pouco utilizado, mas que apresenta correlações positivas com IMC e PC (MAGALHÃES et al., 2014). Estudos em adolescentes brasileiros observaram que o PP correlacionou-se positivamente com homeostasis model of assessment-insulin resistance (HOMA-IR), insulinemia, PA, HDL e TG (GONÇALVES et al., 2014; OLIVEIRA PANI et al., 2015). Apesar da alta prevalência de adolescentes com PP elevado, não foi observada nenhuma associação entre essa medida e marcadores de risco cardiovascular. Alguns fatores podem ser levantados para entender a ausência de associação, como pequeno tamanho da amostra, o qual

Revista Desafios -v. 7, Especial - PIBIC, 2020 
pode ter limitado a eficiência do teste. Outra questão relaciona-se com o ponto de corte utilizado, o mesmo é proveniente de uma população de adolescentes colombianos $($ meninos $=30 \mathrm{~cm}$ e meninas $=29 \mathrm{~cm})$. Apesar disso, posteriormente observamos que esses valores são muito próximos daqueles obtidos em amostras com brasileiros (meninos $=30,4 \mathrm{~cm}$ e meninas $=28,8 \mathrm{~cm})$.

Tabela 3 - Associação entre índices antropométricos e marcadores de risco cardiovascular entre os adolescentes (n=69).

Palmas, TO, Brasil, 2017.

\begin{tabular}{|c|c|c|c|c|c|c|c|c|}
\hline \multirow{2}{*}{$\begin{array}{l}\text { Marcadores de } \\
\text { RCV }\end{array}$} & \multicolumn{2}{|c|}{ IMC } & \multicolumn{2}{|c|}{ PC } & \multicolumn{2}{|c|}{ PP } & \multicolumn{2}{|c|}{ RCE } \\
\hline & $\begin{array}{l}\text { Peso } \\
\text { normal }\end{array}$ & $\begin{array}{l}\text { Excesso } \\
\text { de peso }\end{array}$ & Adequado & Elevado & Adequado & Elevado & Adequado & Elevado \\
\hline \multicolumn{9}{|l|}{ Glicemia* } \\
\hline Adequado (\%) & 97,1 & 100,0 & 97,4 & 100,0 & 100,0 & 97,4 & 97,4 & 100,0 \\
\hline Elevado (\%) & 2,9 & 0,0 & 2,6 & 0,0 & 0,0 & 2,6 & 2,6 & 0,0 \\
\hline \multicolumn{9}{|l|}{ CT* } \\
\hline Adequado (\%) & 77,1 & 37,5 & 71,1 & 60,0 & 25,0 & 74,4 & 71,1 & 60,0 \\
\hline Elevado (\%) & 22,9 & 62,5 & 28,9 & 40,0 & 75,0 & 25,6 & 28,9 & 40,0 \\
\hline Valor $p$ & 0,042 & & 0,630 & & 0,075 & & 0,630 & \\
\hline \multicolumn{9}{|l|}{ LDL* } \\
\hline Adequado (\%) & 80,0 & 75,0 & 78,9 & 80,0 & 50,0 & 82,1 & 78,9 & 80,0 \\
\hline Elevado (\%) & 20,0 & 25,0 & 21,1 & 20,0 & 50,0 & 17,9 & 21,1 & 20,0 \\
\hline Valor $p$ & 1,000 & & 1,000 & & 0,188 & & 1,000 & \\
\hline \multicolumn{9}{|l|}{ HDL* } \\
\hline Adequado (\%) & 55,9 & 62,5 & 54,1 & 80,0 & 100 & 52,6 & 59,5 & 40,0 \\
\hline Elevado (\%) & 44,1 & 37,5 & 45,9 & 20,0 & 0,0 & 47,4 & 40,5 & 60,0 \\
\hline Valor p & 1,000 & & 0,371 & & 0,122 & & 0,636 & \\
\hline \multicolumn{9}{|l|}{ TG* } \\
\hline Adequado (\%) & 71,4 & 57,9 & 73,0 & 40,0 & 50,0 & 71,1 & 70,3 & 60,0 \\
\hline Elevado (\%) & 28,6 & 42,1 & 27,0 & 60,0 & 50,0 & 29,9 & 29,7 & 40,0 \\
\hline Valor $p$ & 0,657 & & 0,162 & & 0,576 & & 0,637 & \\
\hline \multicolumn{9}{|l|}{ PA } \\
\hline Adequado (\%) & 92,5 & 68,8 & 92,9 & 61,5 & 91,7 & 86,0 & 91,7 & 55,6 \\
\hline Elevado (\%) & 7,5 & 31,2 & 7,1 & 38,5 & 8,3 & 14,0 & 8,3 & 44,4 \\
\hline Valor p & $\mathbf{0 , 0 2 6}$ & & 0,009 & & 1,000 & & $\mathbf{0 , 0 1 3}$ & \\
\hline
\end{tabular}

*n= 43. RCV: Risco cardiovascular; IMC: índice de massa corporal; PC: perímetro da cintura; RCE: razão cinturaestatura; PP: perímetro do pescoço; PA: pressão arterial; CT: colesterol total; HDL: lipoproteínas de alta densidade; LDL: lipoproteínas de baixa Densidade; TG: triglicerídeos.

Algumas limitações deste estudo devem ser consideradas, como o fato de 40,2\% da amostra não ter realizado os exames bioquímicos o que diminui o poder dos testes estatísticos em rejeitar a hipótese nula. Outra limitação é a validade externa nos resultados, uma vez que se trata de uma amostra selecionada por conveniência, portanto os resultados aqui deste estudo DOI: http://dx.doi.org/10.20873/uftsuple2020-8844 uso de medidas e índices antropométricos no rastreio de marcadores de risco cardiovascular, sobretudo pela fato da tendência desses marcadores persistirem ao longo da vida, levando ao desenvolvimento de doenças cardiovasculares (MAGNUSSEN et al., 2010). 
utilizou-se a metodologia do erro técnico de medição para avaliação de desempenho dos antropometristas. Aqueles que apresentaram desvios superiores a 1,5\% na análise intra avaliador e a 2,0\% na inter avaliador, passaram por novo treinamento

Destacamos ainda, a questão do estudo ser realizado com adolescentes da região Norte do país, pois sabe-se do escasso número de pesquisas envolvendo essas populações, mesmo existindo particularidades socioeconômicas e culturais importantes a serem consideradas. E por fim, adicionamos que a amostra foi composta somente por adolescentes, sendo estes provenientes das três fases (inicial, intermediária e final). Muitos estudos nessa fase da vida acabam incluindo crianças em suas análises, ou contemplam somente as duas primeiras fases, sendo que a fase final, constantemente é incluída em estudos com adultos.

\section{CONCLUSÃO}

Conclui-se que IMC, PC e RCE estão associados à marcadores de risco cardiovascular na adolescência. Portanto, a antropometria pode ser uma ferramenta útil no rastreamento de indivíduos com risco para o desenvolvimento de doenças cardiovasculares precocemente, tendo em vista a facilidade de obtenção, interpretação e validade. Além disso, os resultados obtidos neste estudo destacam a relevância da antropometria na avaliação nutricional e no acompanhamento da saúde global do adolescente.

\section{AGRADECIMENTO}

Ao Conselho Nacional de Desenvolvimento Científico e Tecnológico (CNPq) e à Coordenação de Aperfeiçoamento de Pessoal de Nível (CAPES) pelo apoio ao estudo

Ao Programa de Iniciação Científica (PIBIC) da UFT pela concessão da bolsa de iniciação científica
À escola que nos recebeu e cedeu todas as condições necessárias para a realização do estudo.

Aos adolescentes e seus pais e/ou responsáveis pela participação e colaboração para que todas as etapas do estudo fossem completadas. Às Secretarias de Educação do Estado do Tocantins e do município de Palmas pelo apoio ao estudo.

Todos os autores declararam não haver qualquer potencial conflito de interesses referente a este artigo.

\section{REFERÊNCIAS}

ANDROUTSOS, O. et al. Neck circumference: A useful screening tool of cardiovascular risk in children. Pediatric Obesity, [S. l.], v. 7, n. 3, p. 187-195, 2012. Disponível em: https://doi.org/10.1111/j.20476310.2012.00052.x

BAUER, K. W. et al. Cardio-metabolic risk screening among adolescents: Understanding the utility of body mass index, waist circumference and waist to height ratio. Pediatric Obesity, [S. l.], v. 10, n. 5, p. 329337, 2015. Disponível em: https://doi.org/10.1111/ijpo.267

BERENSON, G. S. et al. Risk factors in early life as predictors of adult heart disease: The Bogalusa Heart Study. The American Journal od Medical Sciense, [S. l.], v. 298, n. 3, p. 141-151, 1989.

BLOCH, K. V. et al. ERICA: Prevalences of hypertension and obesity in Brazilian adolescents. Revista de Saude Publica, [S. l.], v. 50, n. supl 1, p. 1s-12s, 2016.2 Disponível em: https://doi.org/10.1590/S01518-8787.2016050006685

BROWNING, L. M.; HSIEH, S. D.; ASHWELL, M. A systematic review of waist-to-height ratio as a screening tool for the prediction of cardiovascular disease and diabetes: 05 could be a suitable global boundary value. Nutrition Research Reviews, [S. l.], v. 23, n. 2, p. 247-269, 2010. Disponível em: https://doi.org/10.1017/S0954422410000144

CHOI, D. H. et al. Usefulness of the waist circumference-to-height ratio in screening for obesity and metabolic syndrome among Korean children and adolescents: Korea national health and nutrition examination survey, 2010-2014. Nutrients, [S. l.], v. 9, n. 3, 2017. Disponível em: https://doi.org/10.3390/nu9030256

Revista Desafios -v. 7, Especial - PIBIC, 2020 
FARIA-NETO, J. R. et al. ERICA: Prevalence of dyslipidemia in Brazilian adolescents. Revista de Saude Publica, [S. l.], v. 50, n. supl 1, p. 1s-10s, 2016. Disponível em: https://doi.org/10.1590/S015188787.2016050006723

FERNANDES, R. A. et al. Desempenho de Diferentes Valores Críticos de índice de Massa Corporal $\mathrm{Na}$ Identificação De Excesso De Gordura Corporal E Obesidade Abdominal em Adolescentes. Revista da Associação Médica Brasileira, [S. l.], v. 53, n. 6, p. 515-9, 2007. Disponível em: https://doi.org/10.1590/S0104-42302007000600019

FERREIRA, J. S.; AYDOS, R. D. Prevalence of hypertension among obese children and adolescents | Prevalência de hipertensão arterial em crianças e adolescentes obesos. Ciencia e Saude Coletiva, [S l.], v. 15, n. 1, p. 97-104, 2010.

GOMEZ-ARBELAEZ, D. et al. Neck circumference as a predictor of metabolic syndrome, insulin resistance and low-grade systemic inflammation in children: the ACFIES study. BMC pediatrics, [S. l.], v. 16, p. 31, 2016. Disponível em: https://doi.org/10.1186/s12887-016-0566-1

GONÇALVES, V. S. S. et al. Neck circumference as predictor of excess body fat and cardiovascular risk factors in adolescents. Revista de Nutricao, [S. l.], v. 27, n. 2, p. 161-171, 2014. Disponível em: https://doi.org/10.1590/1415-52732014000200003

JELLIFE, D. B. Evaluacion del estado de nutricion de la comunidad: serie de monografias. [S. l.], p. 191, 1968.

KLEIN, S. et al. Waist Circumference and Cardiometabolic Risk. Diabetes Care, [S. l.], v. 30, n. 6, p. 1647-1652, 2007. Disponível em: https://doi.org/10.2337/dc07-9921

KURTOGLU, S. et al. Neck circumference as a novel parameter to determine metabolic risk factors in obese children. European Journal of Clinical Investigation, [S. l.], v. 42, n. 6, p. 623-630, 2012. Disponível em: https://doi.org/10.1111/j.13652362.2011.02627.x

LO, K. et al. Waist-to-height ratio, body mass index and waist circumference for screening paediatric cardio-metabolic risk factors: a meta-analysis. Obesity Reviews, [S. l.], n. 5, p. 1-18, 2016. Disponível em: https://doi.org/10.1111/obr.12456

MAGALHÃES, E. I. da S. et al. Perímetro da cintura, Relação cintura/estatura e perímetro do pescoço como parâmetros na avaliação da obesidade central em crianças. Revista Paulista de Pediatria, [S. l.], v. 32, n. 3, p. 273-282, 2014. Disponível em: https://doi.org/10.1590/1984-0462201432320

MAGNUSSEN, C. G. et al. Pediatric metabolic syndrome predicts adulthood metabolic syndrome, subclinical atherosclerosis, and type 2 diabetes mellitus but is no better than body mass index alone: The Bogalusa Heart Study and the Cardiovascular Risk in Young Finns Study. Circulation, [S. l.], v. 122, n. 16, p. 1604-1611, 2010. Disponível em: https://doi.org/10.1161/CIRCULATIONAHA.110.94 0809

MOREIRA, C. et al. Ability of different measures of adiposity to identify high metabolic risk in adolescents. Journal of Obesity, [S. l.], v. 2011, 2011. Disponível em: https://doi.org/10.1155/2011/578106

NETO, O. D. A. et al. Fatores associados a' dislipidemia em crianças e adolescentes de escolas públicas de Salvador, Bahia. Revista Brasileira de Epidemiologia, [S. l.], v. 15, n. 2, p. 335-345, 2012. Disponível em: https://doi.org/10.1590/S1415790X2012000200011

OLIVEIRA PANI, O. et al. Co179. Medidas Antropométricas E De Composição Corporal Como Preditores De Risco Cardiovascular E Da Síndrome Metabólica Em Adolescentes. Archivos Latinoamericanos de Nutricion, [S. l.], v. 65, n. 2, p. 259-267, 2015. Disponível em: http://search.proquest.com/openview/800b6b5c805ae 45d78825f51305ab6fb/1.pdf?pqorigsite $=$ gscholar $\&$ cbl $=2032499$

ONIS, M. de et al. Developement of a WHO growth reference for school-aged children and adolescents. Bulletin of the World Health Organization, [S. l.], v. 85, n. February, p. 660-667, 2007. Disponível em: https://doi.org/10.2471/BLT

PAGANO, M. G. K. Princípios de Bioestatística. primeira ed. São Paulo: Cengage Learning, 2008. Ebook.

PEREIRA, P. F. et al. Circunferência da cintura e relação cintura/estatura: Úteis para identifcar risco metabólico em adolescentes do sexo feminino? Revista Paulista de Pediatria, [S. l.], v. 29, n. 3, p. 372-377, 2011. Disponível em: https://doi.org/10.1590/s0103-05822011000300011

PINTO, S. L. et al. Prevalência de pré-hipertensão e de hipertensãoarterial e avaliação de fatores associados em crianças e adolescentes de escolas públicas de Salvador, Bahia, Brasil. Cadernos de Saude Publica, 
[S. l.], v. 27, n. 6, p. 1065-1076, 2011. Disponível em: https://doi.org/10.1590/S0102-311X2011000600004

PREIS, S. R. et al. Neck circumference as a novel measure of cardiometabolic risk: The framingham heart study. Journal of Clinical Endocrinology and Metabolism, [S. l.], v. 95, n. 8, p. 3701-3710, 2010. Disponível em: https://doi.org/10.1210/jc.2009-1779

RASK-MADSEN, C.; KAHN, C. R. Tissue-specific insulin signaling, metabolic syndrome, and cardiovascular disease. Arteriosclerosis, Thrombosis, and Vascular Biology, [S. l.], v. 32, n. 9, p. 2052-2059, 2012. Disponível em: https://doi.org/10.1161/ATVBAHA.111.241919

RIBAS, S. A.; DA SILVA, L. C. S. Fatores de risco cardiovascular e fatores associados em escolares do Município de Belém, Pará, Brasil. Cadernos de Saude Publica, [S. l.], v. 30, n. 3, p. 577-586, 2014. Disponível em: https://doi.org/10.1590/0102$311 \mathrm{X} 00129812$

SILVA, D. A. S. et al. Pressão arterial elevada em adolescentes: Prevalência e fatores associados. Ciencia e Saude Coletiva, [S. l.], v. 18, n. 11, p. 3391-3400, 2013. Disponível em: https://doi.org/10.1590/S1413-81232013001100028

SILVA, K. C. et al. Predictive Ability of Seven Anthropometric Indices for Cardiovascular Risk Markers and Metabolic Syndrome in Adolescents. Journal of Adolescent Health, [S. l.], v. 66, n. 4, p. 491-498, 2020. Disponível em: https://doi.org/10.1016/j.jadohealth.2019.10.021

SISVAN. Orientações básicas para a coleta, processamento, análise de dados e informação em serviços de saúde. [S. l.: s. n.]. E-book. Disponível em: https://doi.org/10.1017/CBO9781107415324.004

SOCIEDADE BRASILEIRA DE CARDIOLOGIA (SBC). Atualização da Diretriz Brasileira de Dislipidemia e Prevenção da Aterosclerose 2017Arquivos Brasileiro de Cardiologia. [S. l.: s. n.].

SOCIEDADE BRASILEIRA DE DIABETES (SBD). Diretriz da Sociedade Brasileira de Diabetes 20172018. [S. l.: s. n.]. Disponível em: https://www.diabetes.org.br/profissionais/images/201 7/diretrizes/diretrizes-sbd-2017-2018.pdf.

STRONG, J. P.; MCGILL, H. C. The pediatric aspects of atherosclerosis. Journal of Atherosclerosis Reseache, [S. l.], v. 9, p. 251-265, 1969.

VIEIRA, M. A. et al. Pressão arterial de crianças e adolescentes de escolas públicas de Cuiabá, Mato Grosso. Acta Paulista de Enfermagem, [S. l.], v. 22, n. spe1, p. 473-475, 2009. Disponível em: https://doi.org/10.1590/s0103-21002009000800002

WANG, Y. et al. Comparison of abdominal adiposity and overall obesity in predicting risk of type 2 diabetes among men. American Journal of Clinical Nutrition, [S. l.], v. 81, n. 3, p. 555-563, 2005. Disponível em: https://doi.org/10.1093/ajen/81.3.555

WHO, W. H. O. Global action plan for the prevention and control of noncommunicable diseases 2013-2020. World Health Organization. [ $S$. l.: s. n.]. Disponível em: https://doi.org/978 924 1506236. 